\title{
Novel Approaches to Unravel Risk Factors and Mechanisms of Venous Thrombosis
}

\author{
Inga Scheller ${ }^{1,2}$ Bernhard Nieswandt ${ }^{1,2}$ \\ ${ }^{1}$ Institute of Experimental Biomedicine I, University Hospital, \\ University of Würzburg, Würzburg, Germany \\ 2 Rudolf Virchow Center, University of Würzburg, Würzburg, Germany
}

Thromb Haemost 2020;120:372.

Venous thrombosis (VT) is a leading cause of mortality and morbidity in industrialized countries. However, the precise mechanisms that trigger clotting in large veins are not fully understood. Many genetic and acquired risk factors have been identified for VT that alter blood flow, activate the endothelium, and alter the activity of coagulation factors. ${ }^{1}$ Many of these clotting factors are localized in the plasma, making analysis of this blood compartment highly interesting for the understanding of VT.

As an alternative to activity- or antibody-based plasma protein assays, which provide only limited information on single proteins in a sample, mass spectrometry (MS)-based proteomics allows high-throughput, quantitative analysis of biomolecules. Indeed, quantitative MS has been successfully applied to understand the pathomechanisms of multiple diseases. ${ }^{2}$

In this issue of Thrombosis and Haemostasis, Tilburg et al attempt to uncover the plasma protein signatures upon induction of VT using state-of-the-art MS-based targeted proteomics in combination with a murine knockout model for SLC44A2, a recently identified susceptibility locus for VT. $^{3,4}$ With this elegant approach, they not only gain information about changes in the plasma proteome in VT, but also demonstrate the great advantages of MS-based proteomics such as specificity and the possibility to expand a limited sample volume to a huge dataset.

The authors could show that the experimental induction of VT induced changes in plasma levels of multiple proteins,
Address for correspondence Prof. Dr., Bernhard Nieswandt, Institute of Experimental Biomedicine I, University Hospital, University of Würzburg, 97080 Würzburg, Germany

(e-mail: bernhard.nieswandt@virchow.uni-wuerzburg.de).

including acute phase proteins as well as proteins related to erythrocyte function. Strikingly, a strong sex-dependency of these changes was observed that even overruled the effects of SLC44A2 deficiency.

By clarifying the plasma protein signatures in VT, the present study not only advances our understanding of cardiovascular diseases but also introduces a new technique to diagnose such pathologies, identify new targets for therapeutic interventions, and quantify the effects of existing immunotherapeutics.

Conflict of Interest

None declared.

\section{References}

1 Mackman N. New insights into the mechanisms of venous thrombosis. J Clinical Investigation 2012. Doi: 10.1172/JCI60229

2 Geyer PE, Kulak NA, Pichler G, Holdt LM, Teupser D, Mann M. Plasma proteome profiling to assess human health and disease. Cell Syst 2016;2(03):185-195

3 Tilburg J, Michaud SA, Maracle CX, et al. Plasma protein signatures of a murine venous thrombosis model and Slc44a2 knockout mice using quantitative-targeted proteomics. Thromb Haemost 2020; 120(03):423-436

4 Germain M, Chasman DI, de Haan H, et al; Cardiogenics Consortium. Meta-analysis of 65,734 individuals identifies TSPAN15 and SLC44A2 as two susceptibility loci for venous thromboembolism. Am J Hum Genet 2015;96(04):532-542 received

January 28, 2020

accepted

January 28, 2020 (c) 2020 Georg Thieme Verlag KG

Stuttgart · New York
DOI https://doi.org/

10.1055/s-0040-1702232. ISSN 0340-6245. 